\title{
Postoperative Analgesia by Transmuscular Quadratus Lumborum Block Catheters
}

\author{
Ashok Jadon ${ }^{1 *}$, Mayur Motka ${ }^{2}$, Asit Kumar Pati ${ }^{3}$ and Neelam Sinha ${ }^{4}$ \\ ${ }^{1}$ Chief Consultant \& Head of Department, India \\ ${ }^{2}$ DNB student, India \\ ${ }^{3}$ Specialist, India \\ ${ }^{4}$ Specialist, India
}

Submission: November 21, 2016; Published: December 01, 2016

*Corresponding author: Ashok Jadon, Chief Consultant \& Head of Department, Duplex-63, Vijaya Heritage Phase-6, Kadma, Jamshedpur-831005 Jharkhand, India, Tel: +91-9234554341; Email: jadona@rediffmail.com

\begin{abstract}
Ultrasound guided Quadratus lumborum (QL) block is a relatively new regional anesthetic technique which provides effective postoperative analgesia for abdominal surgeries as a part of multimodal analgesic regimen. We report a case of hysterectomy where effective postoperative analgesia was provided by ultrasound guided bilateral QL-3 block and infusion of local anesthetic through catheters inserted postoperatively.
\end{abstract}

Keywords: Local anaesthetic; ropivacaine; Postoperative analgesia; Quadratus lumborum blocks; type-1, 2 and transmuscular (type-3); Truncal blocks; Ultrasound guided blocks

\section{Introduction}

Postoperative analgesia is an integral part of successful surgical outcome. Epidural analgesia with catheter technique remains the most effective analgesic technique after abdominal surgery. However, it has limitation in patients who are either on anticoagulants and have cardiac morbidity due to significant hemodynamic changes which may be detrimental to such patients $[1,2]$. Ultrasound guided Quadratus lumborum block (QLB) block is a new technique of regional analgesia [3,4]. There have been reports of excellent post-operative pain management with the QLB for both abdominal and retroperitoneal surgery [5,6]. However, postoperative analgesia by bilateral transmuscular quadratus lumborum blocks (TQLB) or QL-3 block with catheter is rarely reported. We present a case report where postoperative analgesia was provided by bilateral transmuscular quadratus lumborum blocks (TQLB) and infusion of local anaesthetics through inserting catheters ina patient who had cardiac disease and underwent hysterectomy operation.

\section{Case}

A female aged 58yrs posted for abdominal hysterectomy with bilateral oophorectomy for dysfunctional uterine bleeding and fibroid. She had coexisting morbidities of rheumatic heart diseases (mitral valve replacement in 1983 for mitral stenosis), hemiplegia of left side 1990 (completely recovered), diabetes mellitus, hypertension, persistent chronic atrial fibrillation and pulmonary hypertension. Preoperative 2D echocardiography showed moderately stenotic prosthetic valve, moderate tricuspid regurgitation, mild mitral regurgitation, and mild aortic regurgitation, dilated left atrium, pulmonary hypertension and left ventricular ejection fraction $40 \%$. X-rays showed Lower Zone haziness. Her blood pressure was 160/70 mm Hg, pulse 98/minute irregular. On auscultation diastolic murmur with metallic clickof prosthetic mitral valve was present. She was on oral warfarin which was changed to unfractionated heparin 5000 International units twice daily 5 days before the day of surgery. She was also taking antihypertensive, digoxin, oral hypoglycemic and human act rapid insulin. Laboratory investigations regarding haemogram, coagulation profile and other biochemical parameters were within normal limits. An informed consent about anaesthesia and postoperative analgesia plan was taken. After overnight fasting on the day of surgery all oral medicines and intravenous prophylactic antibiotics were given. Blood sugar levels were monitored 6hrly and insulin dosage were used according to sugar levels. 
In operation theatre non-invasive monitoring (ECG, Oximeter, Noninvasive Blood Pressure) were attached.Under local anaesthesia and using ultrasound guidanceright sided internal juglar central venous line was inserted (Linear 6-13 MHz M-Turbo ${ }^{\circledR}$ SonoSite ultrasound system) taking all aseptic measures. General anaesthesia was induced with intravenous fentanyl $(100 \mu \mathrm{gm})$ and $100 \mathrm{mg}$ propofol. A size\#3 proseal LMA was inserted and anaesthesia was maintained with $1 \%$ isoflurane, $50-50 \%$ oxygen-nitrous mixture and vecuronium for muscle relaxation. Operative course was uneventful except patient required positive end expiratory pressure (PEEP) of 6 to maintain saturation above $95 \%$.

After the surgery bilateral ultrasound guided transmuscular quadrates lumborum (TQL) block was given using curved array transducer 5-2 MHz (M-Turbo® SonoSite ultrasound system). In lateral position, the side to be blocked was kept up and probe was placed in the midaxillary line in the transverse plane immediately above the iliac crest and then it was slided dorsally till the Shamrock sign was clearly identified [7]. In "Shamrock sign" The quadratus lumborum (QL) muscle is seen as a superior leaf of the Shamrock at the apex of the transverse process (TP) of L4, erector spinae (ES) muscles make up the posterior leaf, psoas major (PM) muscle makes the anterior leaf and the transverse process (TP)represents the stem connecting the 3leaves (Figure 1). 18 G Tuohy needle was inserted from the posterior end of the probe and directed for the fascial plane between the QL and PM muscles through the QL muscle (Figure 2). Once needle was confirmed at correct location $25 \mathrm{~mL} 0.3 \%$ ropivacaine was injected [8](Figure 3) and 18G epidural catheter was inserted $3 \mathrm{~cm}$ beyond the tip and fixed at skin through subcutaneous tunnel. Other side catheter was also inserted in similar manner. Catheter position was confirmed by injection of local anaesthetic and using color doppler mode (Figure 4). After reversal patient was shifted to recovery room. Both the catheters were connected together using a y-connection to a volumetric pump and infusion of ropivacaine $0.1 \%$ and fentanyl $1 \mu \mathrm{g} / \mathrm{mL}$ was started @ 14ml/ hour. Additional analgesia was provided by injection paracetamol $1 \mathrm{G}$ intravenous $8 \mathrm{hrly}$ for first 48 hours and then 12 hourly for next two days. Injection Tramadol $50 \mathrm{mg}$ IV as an when required was advised for rescue analgesia. Pain scores using Visual Analogue Scale (VAS)were monitored two hourly for 24 hours and then 4 hrly for next 2 days. VAS score remain $0-2$ on rest (where $0=$ no pain and 10=maximum pain) and $0-4$ on movement for 3 days. No rescue analgesia was required. Patient also reported high satisfaction from this technique of pain relief compared to two previous caesarean section surgery.A contrast study was done with injection of $5 \mathrm{~mL}$ contrast (Omnipaque-300®) mixed with $15 \mathrm{~mL} 0.3 \%$ ropivacaine which showed spread up to the thoracic paravertebral space (Figure 5). Catheters were removed after 3days, heparin was tapered and oral anticoagulant was started. On $5^{\text {th }}$ day patient was discharged home after check dressing.

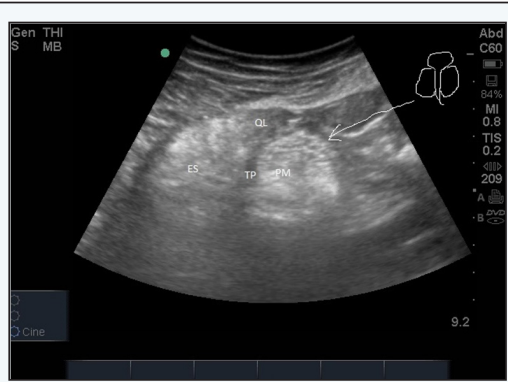

Figure 1: "Shamrock sign" The quadratus lumborum (QL) muscle is seen as a superior leaf of the Shamrock at the apex of the transverse process (TP) of L4, erector spinae (ES) muscles make up the posterior leaf, psoas major (PM) muscle makes the anterior leaf and the transverse process (TP) represents the stem connecting the 3 leaves.

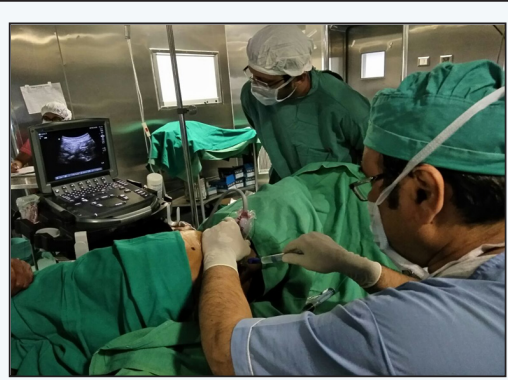

Figure 2: $18 \mathrm{G}$ Tuohy needle was inserted from the posterior end of the probe and directed for the fascial plane between the $\mathrm{QL}$ and PM muscles through the QL muscle.
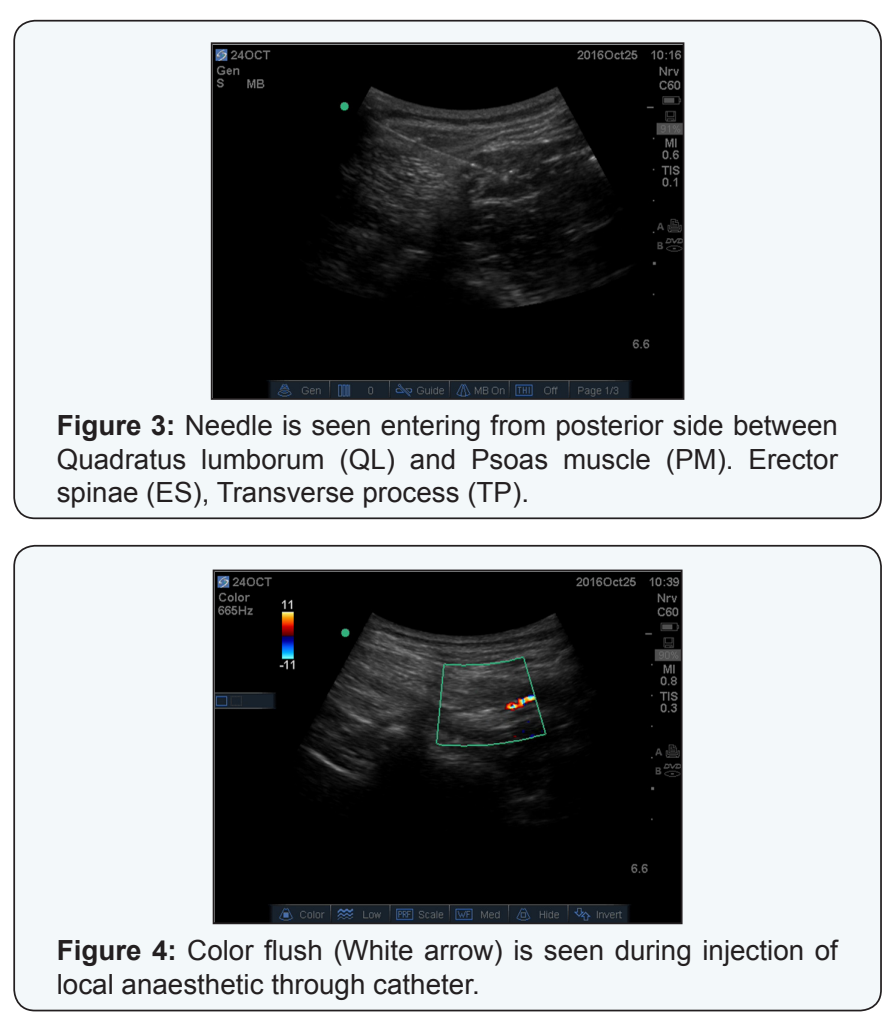


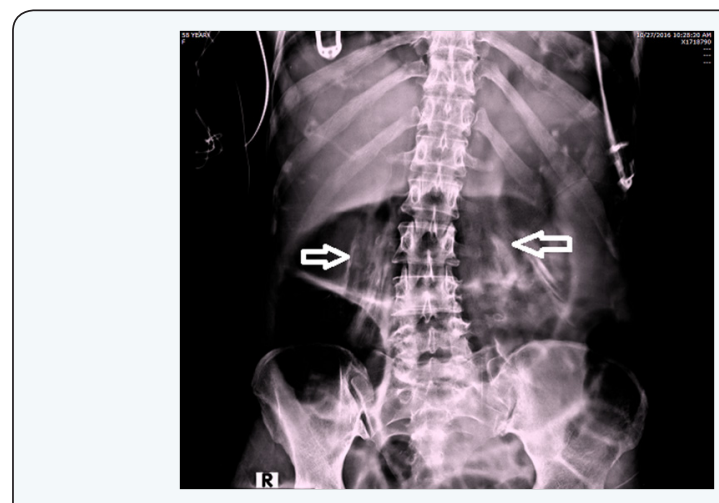

Figure-5: Postoperative X-ray (Anterior-Posterior View) after contrast injection showing bilateral contrast spread close to spine (paravertebral space)

\section{Discussion}

QL block was described by Blanco where injection was given anterior to the QL (QL block type-1) and later he modified his approach by injecting local anesthetic posterior to QL (QL block type-2) [9]. He postulated that, injection of local anasthetic in the facial plane over QL results in extended spread up to thoracic dermatomes. Carney et al documented through contrast enhancement from T4 to L2 that, the QL block as an extension of the local anaesthetic beyond the TAP-plane spreading into the thoracic paravertebral space with the provision of both visceral and somatosensory blockade of the abdominal wall [10]. Kadam RV [11] observed the sensory spread from T8 toL1 in a case of laparotomy done for tumor excision and have suggested that sensory analgesia is variable and dependent on many factors including the volume of the drug and site of injection.

Single injection QL block has been used successfully for the management of postoperative pain relief after caesarean section [12] for sub-total gastrectomy in a septic patient [13], laparotomy for duodenal tumour excision and to manage chronic back pain [14]. The effective pain relief lasted for 12-14hours. Prolonged and continuous pain relief has been reported with catheter techniques for acute abdominal pain management [15] breast reconstruction [16] and laparotomy [11]. However, they all have used type-2 quadratus lumborum block.

In our case we used a transmuscular approach (QL block type3) suggested by Borglum et al. [4] where the local anaesthetic is injected in the fascial plane between the QL and Psoas Major (PM) muscles. This technique has more definitive endpoint of injection and injection into this fascial plane ensured a reliable spread in to the thoracic paravertebral space (TPVS) [4].The presence of psoas muscle ahead of needle point also works as safeguard to prevent inadvertent entry in to the peritoneal space and injury to kidneys.

Transmuscular quadratus lumborum or QL type-3 block has been used successfully for pain management after caesarean section [17]. Where 14 hours of effective analgesia was provide by this block. QL type-3 with catheter has been used effectively to manage pain of hip arthroplasty in place of epidural analgesia
[18]. To our understanding bilateral catheter technique to provide continuous analgesia is not reported earlier.

Epidural was an option in our case but on evaluation a relative risk of serious complication we preferred this technique $[1,2]$.

We could achieve effective analgesia by insertion of bilateral catheter to control visceral as well as somatic pain postoperatively. However, it would have been interesting to observe analgesia influence on intraoperative hemodynamics if, catheter would have been inserted preoperatively.

The unanswered question remains that, which QL block is more effective? The efficacy of QL block is correlated with spread of local anesthetic up to the paravertebral space and we have experienced (unpublished data) that posterior spread of QL-3 is more consistent than QL-1 and QL-2. This happens because fascia transversalis (FT) covers the thoracolumbar fascia (TLF), anterior surface of the QL as well as the antero-lateral surface of the PM [19]. The FT splits in two sheets at the level of the diaphragm. One sheet becomes the inferior diaphragmatic fascia and the other sheet passes behind the lateral and medial arcuate ligaments and continue as endothoracic fascia [20]. Thus, LA injected in the lumbar region between the PM and QL can spread up to TPVS [21].

However, there has been no comparative study to suggest one's superiority over other for safety and efficacy.

Although, QL-3 seems more invasive than QL-2 however, entry through QL muscle and presence of psoas as a guard in front of needle make it as a safer choice.

Contrary to QL-1 and QL-2, which can be given in supine or wedge under hip position. QL-3 requires a lateral decubitus position and become the major limitation for the procedure even if used preoperatively because, extra helping hands are required.

We monitored the clinical effect in terms of pain relief however, sensory spread was not recorded. We feel it was a miss on our part as it would has helped further studies. This we consider a limitation of our case report.

\section{Conclusion}

In this case report we observed that QL-3 block is an effective technique for postoperative pain relief after hysterectomy surgery in a high risk patient. Infusion of local anaesthetic through catheters inserted during block further helped in continuous pain relief for desired period. We did not face any complication however, more studies are required to prove its efficacy and safety in high risk patients.

\section{References}

1. Macintyre PE, Scott DA, Schug SA, Visser EJ, Walker SM (2010) Acute Pain Management: Scientific Evidence. ( $3^{\text {rd }}$ edn), Australia and New Zealand College of Anaesthetists and Faculty of Pain Medicine 175224.

2. Rawal N (2012) Epidural technique for postoperative pain: gold standard nomore? Reg Anesth Pain Med 37(3): 310-317. 
3. Blanco R (2007) Tap block under ultrasound guidance: the description of a "no pops" technique. Reg Anesth Pain Med 32(5): 130.

4. Borglum J, Moriggl B, Jensen K, Lonnqvist PA, Christensen AF, et al. (2013) Ultrasound-guided Transmuscular Quadratus Lumborum Blockade. Br J Anaesth.

5. Chakraborty A, Goswami J, Patro V (2015) Ultrasound-Guided Continuous Quadratus Lumborum Block for Postoperative Analgesia in a Pediatric Patient. A A Case Rep 4(3): 34-36.

6. Visoiu M, Yakovleva N (2013) Continuous postoperative analgesia viaquadratus lumborum block - An alternative to transversus abdominis plane block. Paediatr Anaesth 23(10): 959-961.

7. Sauter AR, Ullensvang K, Niemi G, Lorentzen HT, Bendtsen TF, et al. (2015) The Shamrock lumbar plexus block: A dose-finding study. Eur Anaesthesiol 32(11): 764-770.

8. Rosenberg PH, Veering BT, Urmey WF (2004) Maximum recommendeddoses of local anesthetics: A multifactorial concept. Reg Anesth Pain Med 29(6): 564-575.

9. Blanco R (2014) Optimal point of injection: the quadratum lumborumtype I and II blocks. Anaesthesia 68(4).

10. Carney J, Finnerty O, Rauf J, Bergin D, Laffey JG, et al. (2011) Studies on the spread of local anaesthetic solution in transversus abdominis plane blocks. Anaesthesia 66(11): 1023-1030.

11. Kadam RV (2013) Ultrasoundguided quadratus lumborum block as a postoperative analgesic technique for laparotomy. J Anaesthesiol Clin Pharmacol 29(4): 550-552.

12. Blanco R, Ansari T, Girgis E (2015) Quadratus lumborum block forpostoperative pain after caesarean section: A randomised controlled trial. Eur J Anaesthesiol 32(11): 812-818.
13. Cardoso JM, Sa M, Reis H, Almeida L, Sampaio JC, Pinheiro C, et al (2016) Type II Quadratus Lumborum block for a sub-total gastrectomy in a septic patient. Rev Bras Anestesiol 26.

14. Carvalho R (2016) Bloqueio do quadradolombaremdorcrônicapóshernioplastia abdominal: relato de caso. Rev Bras Anestesiol.

15. Shaaban M, Esa WA, Maheshwari K, Elsharkawy H, Soliman LM (2015) Bilateral continuous quadratus lumborum block for acute postoperative abdominal pain as a rescue after opioid induced respiratory depression. A A Case Rep 5(7): 107-111.

16. Spence NZ, Olszynski, P, Lehan A, Horn JL, Webb CA (2016) Quadratus lumborum catheters for breastreconstruction requiring transverse rectus abdominis myocutaneous flaps. J Anesth 30(3): 506-509.

17. Maenchen N, Hansen CK, Dam M, Borglum J, Mette Dam (2016) Ultrasound-guided Transmuscular Quadratus Lumborum (TQL) Block for Pain Management after Caesarean Section. Int J Anesthetic Anesthesiol 3(2): 048.

18. Ueshima H, Yoshiyama S, Otake H (2016) The ultrasound-guided continuous transmuscular quadratus lumborum block is an effective analgesia for totalhip arthroplasty. J Clin Anesth 31: 35.

19. Hollinshed WH (1971) The abdominal Wall and Inguinal Region. In: Hollinshed WH, Paul B Hoeber (Eds.), Anatomy for surgeons ( $\left.2^{\text {nd }} \mathrm{edn}\right)$, The Thorax, Abdomen and Pelvis, New York, USA, pp. 220-222.

20. Karmakar MK, Gin T, Ho AM-H (2001) Ipsilateral thoraco-lumbar anaesthesia and paravertebral spread after low thoracic paravertebral injection. Br J Anaesth 87(2): 312-316

21. Williams PL, Warwick R, Dyson M, Bannister LH (1989) Fasciae and Muscles of the Trunk; Muscles of the Lower Limb. In: Williams PL, Warwick R, et al. (Eds.), Gray's Anatomy (37 th $e d n)$, Churchill Livingstone, Edinburgh, London, pp. 604-635.

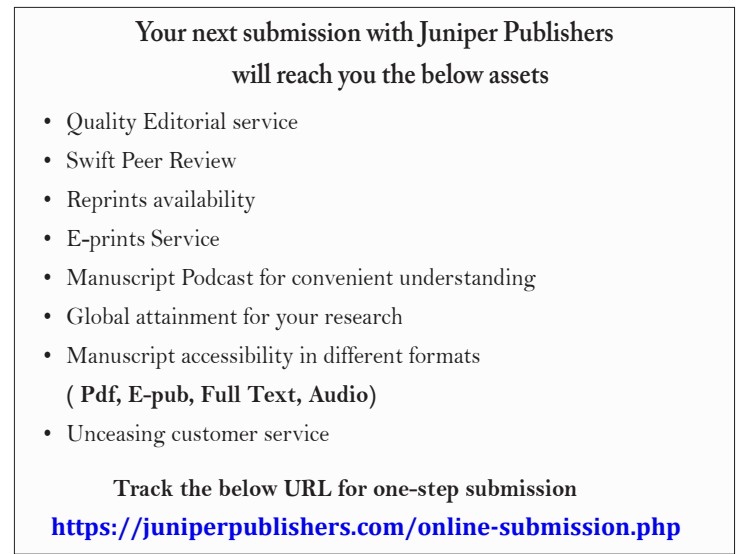

